\title{
Pre-pregnancy body mass index, height and physical activity are associated with rate of gestational weight gain among Malaysian mothers
}

\begin{abstract}
Aim: Both inadequate and excessive weight gain during pregnancy can have immediate and long-term health risks for women and infants. This study investigated rate of gestational weight gain (GWG) and its associated factors in Malaysian pregnant women. Methods: This cross-sectional study was conducted at maternal and child health clinics in Selangor and Negeri Sembilan between November 2010 and April 2012. A pre-tested questionnaire was used to obtain sociodemographic, obstetric, dietary intake and physical activity information. Current weight and height were measured using standard procedures. GWG rate was calculated as the average weekly weight gain in that particular trimester of pregnancy and further categorized according to the Institute of Medicine (IOM) recommendations. Results: Mean GWG rate for all pre-pregnancy BMI categories in the second and third trimesters was higher than the IOM recommendations. Overweight women (adjusted OR, 4.26; 95\% CI: 1.92-9.44) and women $<153 \mathrm{~cm}$ tall (adjusted OR, 1.96; 95\%CI: 1.21-3.18) tend to have inadequate GWG rate. Women with high pre-pregnancy body mass index (BMI; $\geq 25.0$ $\mathrm{kg} / \mathrm{m}^{2}$; overweight: adjusted OR, 3.88; 95\%CI: 2.12-7.09; obese: adjusted OR, 2.34; 95\%CI: 1.28-4.29) and low physical activity (adjusted OR, 1.74; 95\%CI: 0.77-3.97) were twothreefold more likely to have excessive GWG. Conclusion: Both inadequate and excessive GWG can have detrimental effects on the health of mothers and infants. Pre-pregnancy BMI, height and physical activity should be emphasized in prenatal care to ensure that women have adequate GWG rate.
\end{abstract}

Keyword: Height; Physical activity; Pre-pregnancy body mass index; Rate of gestational weight gain 